\title{
Unidentified quasars among stationary objects from Gaia DR2
}

\author{
K. E. Heintz ${ }^{1,2}$, J. P. U. Fynbo ${ }^{2}$, E. Høg ${ }^{3}$, P. Møller ${ }^{4}$, J.-K. Krogager ${ }^{5}$, S. Geier ${ }^{6,7}$ P. Jakobsson ${ }^{1}$, and L. Christensen ${ }^{8}$ \\ ${ }^{1}$ Centre for Astrophysics and Cosmology, Science Institute, University of Iceland, Dunhagi 5, 107 Reykjavík, Iceland \\ e-mail: keh14@hi.is \\ 2 The Cosmic Dawn Center, Niels Bohr Institute, University of Copenhagen, Juliane Maries Vej 30, 2100 Copenhagen $\emptyset$, Denmark \\ e-mail: heintz@dark-cosmology.dk \\ 3 Niels Bohr Institute, University of Copenhagen, Juliane Maries Vej 30, 2100 Copenhagen Ø, Denmark \\ ${ }^{4}$ European Southern Observatory, Karl-Schwarzschildstrasse 2, 85748 Garching bei München, Germany \\ 5 Institut d'Astrophysique de Paris, CNRS-UPMC, UMR7095, 98bis bd Arago, 75014 Paris, France \\ ${ }^{6}$ Gran Telescopio Canarias (GRANTECAN), Cuesta de San José s/n, 38712 Breña Baja, La Palma, Spain \\ 7 Instituto de Astrofísica de Canarias, Vía Láctea s/n, 38200 La Laguna, Tenerife, Spain \\ 8 Dark Cosmology Centre, Niels Bohr Institute, University of Copenhagen, Juliane Maries Vej 30, 2100 Copenhagen $\emptyset$, Denmark
}

Received 8 May 2018 / Accepted 22 June 2018

\begin{abstract}
Here we apply a technique selecting quasar candidates purely as sources with zero proper motions in the Gaia data release 2 (DR2). We demonstrate that this approach is highly efficient toward high Galactic latitudes with $\$ 25 \%$ contamination from stellar sources. Such a selection technique offers very pure sample completeness, since all cosmological point sources are selected regardless of their intrinsic spectral properties within the limiting magnitude of Gaia. We carry out a pilot-study, defining a sample compiled by including all Gaia-DR2 sources within one degree of the north Galactic pole (NGP) selected to have proper motions consistent with zero within $2 \sigma$ uncertainty. By cross-matching the sample to the optical Sloan Digital Sky Survey (SDSS) and the mid-infrared (MIR) AllWISE photometric catalogues, we investigate the colours of each of our sources. We determine the efficiency of our selection by comparison with previously spectroscopically confirmed quasars. The majority of the zero-proper-motion sources selected here have optical to MIR colours consistent with known quasars. The remaining population may be contaminating stellar sources, but some may also be quasars with colours similar to stars. Spectroscopic follow-up of the zero-proper-motion sources is needed to unveil such a hitherto hidden quasar population. This approach has the potential to allow substantial progress on many important questions concerning quasars, such as determining the fraction of dust-obscured quasars, the fraction of broad absorption line (BAL) quasars, and the metallicity distribution of damped Lyman- $\alpha$ absorbers. The technique could also potentially reveal new types of quasars or even new classes of cosmological point sources.
\end{abstract}

Key words. astrometry - proper motions - quasars: general

\section{Introduction}

There is great interest in building unbiased catalogues of quasars for use in answering a range of important astrophysical questions. Further constraints on these objects are needed to further our understanding of the quasar phenomenon itself and the growth and occurrence of supermassive black holes through cosmic time. Quasars are also used as probes of intervening material, and have been shown to play a role in re-ionisation of both hydrogen and helium, and effect the ultraviolet (UV) background levels throughout the universe (Hewett \& Foltz 1994; Weymann et al. 1981). Most current quasar surveys, however, rely on their specific intrinsic properties, such as strong UV emission (Schneider et al. 2010), distinct near-infrared (NIR) and mid-infrared (MIR) colours (Maddox et al. 2012; Secrest et al. 2015), X-ray output Brusa et al. (2010) or prominent radio emission (Ivezić et al. 2002).

In this Letter we apply an astrometric approach, identifying quasars as apparently stationary sources on the sky, based purely on the astrometric measurement from the Gaia mission (Heintz et al. 2015), and present a pilot study. Such a technique was already proposed by Koo et al. (1986) but has so far not been widely applied. Our goal here is to quantify the efficiency and completeness of this selection technique. Identifying quasars based only on their zero proper motions has the potential to open a route of selecting quasars in an unbiased way, and might even lead to the discovery of new types of quasars or other types of extragalactic point sources.

\section{Astrometric selection of quasars}

The Gaia data release 2 (DR2; Gaia Collaboration 2018) catalogue consists of more than $1.3 \times 10^{9}$ sources down to $G \approx 21 \mathrm{mag}$, for which the five-parameter astrometric solution (positions, parallaxes, and proper motions) has been determined (Lindegren et al. 2018). The Gaia $G$ filter is very broad, covering the spectral range from 400 to $1000 \mathrm{~nm}$, and therefore quasars in a wide range of redshifts should be included in the catalogue.

We extract all sources within a radius of one degree from the north Galactic pole (NGP) centred on $(\alpha, \delta)=$ $12^{\mathrm{h}} 51^{\mathrm{m}} 26^{\mathrm{s}} .0+27^{\circ} 07^{\mathrm{m}} 42^{\mathrm{ps}} .0$ from the Gaia DR2 catalogue (see Fig. 1). We then limit our search to sources with $18<G<20 \mathrm{mag}$, for which the associated uncertainty is up to $1.2 \mathrm{mas} \mathrm{yr}^{-1}$ in the respective proper motion components. This is motivated by our pre-study (Heintz et al. 2015) in which we (based on pre-launch 


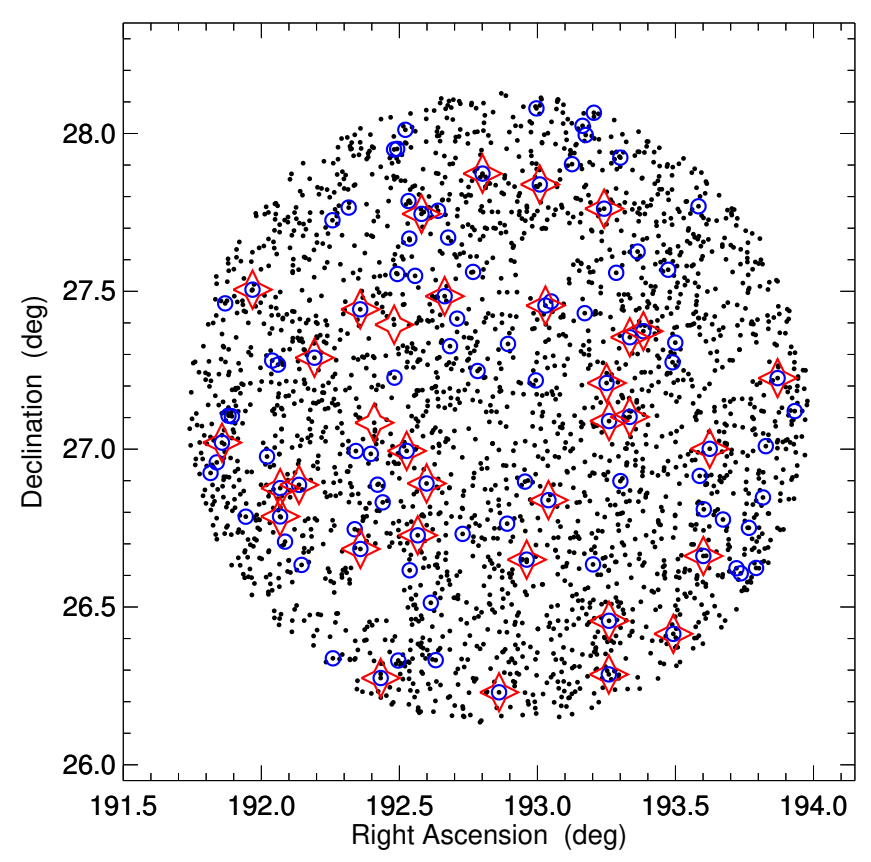

Fig. 1. Location on the sky of all point-like Gaia sources with proper motions and $18<G<20$ mag (black dots) within one degree of the NGP. The subset of these with proper motions consistent with zero within $2 \sigma$ are shown by the blue circles and those that are already spectroscopically confirmed quasars are shown by the red star symbols.

simulations of the Gaia data) found that the expected contamination of apparently stationary stars is lower than $\approx 20 \%$ at the Galactic poles, but increases significantly when observing closer to the Galactic plane or at magnitudes brighter than $G<18$ mag. This criterion also rejects the faintest Gaia sources $(G>20 \mathrm{mag})$, which still have significant errors on their measured proper motions. We then select all point sources with total proper motions, $\mu=\sqrt{\mu_{\mathrm{RA}}^{2}+\mu_{\mathrm{Dec}}^{2}}$, consistent with zero at the $2 \sigma$ confidence level (i.e. $S / N_{\mu}=\mu / \mu_{\text {err }}<2$ ). Finally, we identify the counterpart to each source in the Sloan Digital Sky Survey (SDSS) and require that all Gaia sources have morphologies consistent with being point sources (class $=6$ in the SDSS) to limit our search to quasars only (i.e. excluding Seyferts and potential contaminating extended galaxies). This results in about $2 \%$ of the sample being removed due to extended morphology. Matching the Gaia sample to the SDSS with a matching radius of less than 1 arcsec also allows us to investigate the properties of our sample in optical colour-colour space.

In total, we find that there are 2634 point sources observed by Gaia, all with measured proper motions and $18<G<20$ mag, within one degree of the NGP, of which 100 sources $(\approx 4 \%)$ have proper motions consistent with zero (within $2 \sigma$ ). These are marked with blue circles in Fig. 1. Cross-matching our extracted catalogue with the SDSS data release 14 quasar sample (SDSS-DR14Q, Pâris et al. 2018) and the NASA/IPAC Extragalactic Database (NED), we find that 34 quasars are already spectroscopically confirmed within the same magnitude limit and region on the sky, of which $32(\approx 95 \%)$ also have $S / N_{\mu}=\mu / \mu_{\mathrm{err}}<2$. This number is expected based on the statistics of the $2 \sigma$ cut. For the remaining two, the measured proper

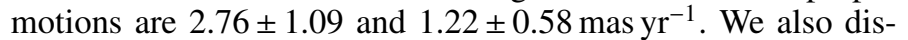
cover two spectroscopically confirmed stars, observed as part of the SDSS-APOGEE survey (Alam et al. 2015). An extract

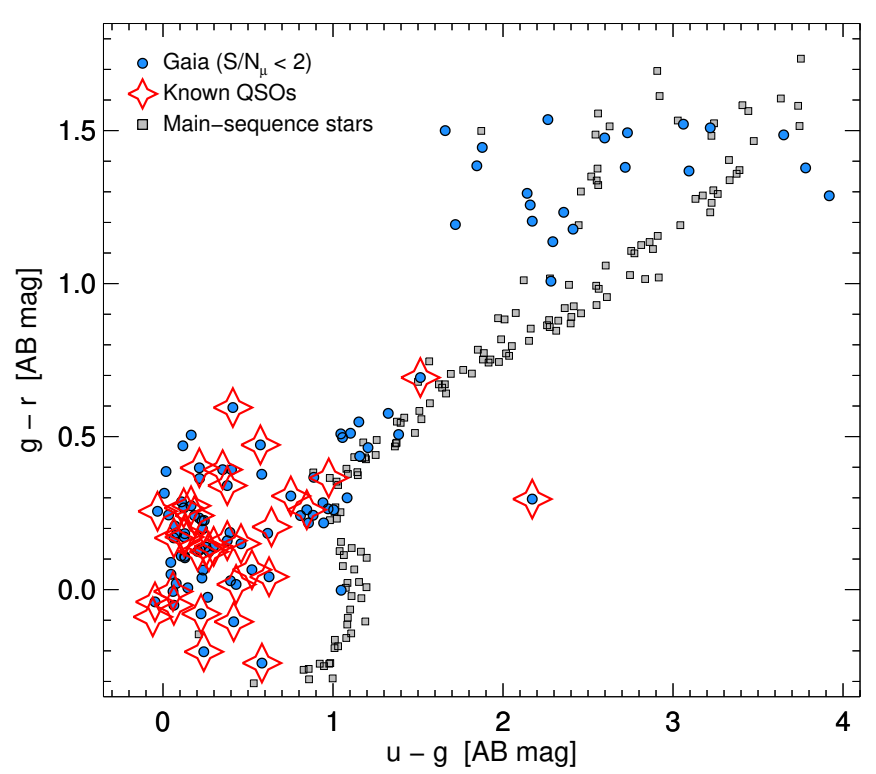

Fig. 2. Optical colour-colour plots of the WISE-detected Gaia point sources with proper motions and $18<G<20$ mag (black dots) within one degree of the NGP. Gaia point sources with zero proper motions are represented by the blue dots and the spectroscopically confirmed quasars are shown by the red star symbols. Typical stellar colours are shown as grey dots.

of the full sample of Gaia sources with zero proper motions is presented in Table A.1. We also examine the additional requirement that the sources have parallaxes consistent with zero within $3 \sigma$, but only five sources (GQs $1255+2707,1248+2658$, $1247+2655,1247+2706$, and $1251+2804)$ were outside this criterion so we chose to include them for completeness.

\section{Selection efficiency and completeness}

We now investigate the location of the Gaia sources with zero proper motions in optical colour-colour space. By doing so, we can examine whether these candidate quasars have, for example, UV excess typical of unobscured, low- $z$ quasars (e.g. Sandage 1965 ; Schmidt \& Green 1983). About $70 \%$ of the zero proper motion sources have blue $(u-g<1)$ colours (see Fig. 2). For quasars at $z \gtrsim 2.2$, the Lyman- $\alpha$ emission line will move out of the $u$ band, such that the quasars appear redder in $u-g$ colour space. At red $g-r$ colours $(g-r>1)$, the zero-proper-motion sources have optical colours consistent with $\mathrm{M}$ or $\mathrm{G}$ dwarf stars. While some of these are likely to be stellar contaminations, removing these candidates will also exclude dust-reddened quasars and broad absorption line (BAL) quasars from the sample, which are found to have very red optical colours and to be systematically missing in most existing quasar samples (Fynbo et al. 2013; Krogager et al. 2015, 2016; Ross et al. 2015; Krawczyk et al. 2015).

To assess the efficiency of our selection, we cross-match our sample of Gaia sources with zero proper motions to the all-sky MIR survey based on the WISE satellite (AllWISE; Wright et al. 2010). Mid-infrared selection of quasars is efficient at separating stars and galaxies from quasars and is not affected by dust extinction, while also being sensitive to high-redshift quasars. Of the 100 Gaia point sources with zero proper motions, we identify 76 of the counterparts in the AllWISE catalogue within 1 arcsec. This cross-match might introduce a bias excluding quasars with weak IR emission. Stellar contaminants will also 


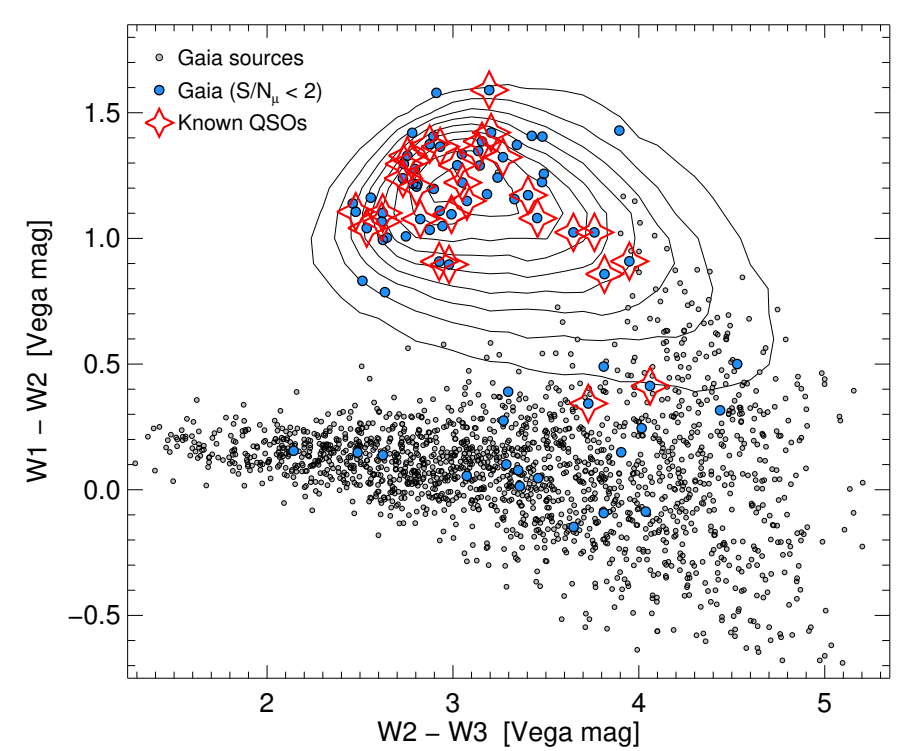

Fig. 3. WISE colour-colour plot of all Gaia point sources (black dots), the subset with zero proper motions (blue dots), and spectroscopically confirmed quasars (red star symbols) within one degree of the NGP. Overplotted are contour levels linearly spaced in ten steps from 0 to $100 \%$ of the full SDSS-DR14 quasar sample with MIR counterparts in the AllWISE catalogue.

have weak IR emission however, and we find that of the 24 sources excluded in this approach, roughly half have a significant UV excess, whereas the other half have optical colours consistent with the main sequence stellar track. In Fig. 3, we show the zero proper motion Gaia sources in mid-infrared colour-colour space. Overplotted are contours of the SDSS-DR14Q sample for which WISE photometry exists. A simple colour criterion of $W 1-W 2>0.8$ has been found to be robust in identifying quasars at most redshifts (Stern et al. 2012). In our sample of zero proper motion sources with WISE photometry, $55(70 \%)$ have $W 1-W 2>0.8$ (of which 29 are already identified quasars) and have WISE colours consistent with the full SDSS-DR14Q sample. We consider the remaining 26 sources as high-likelihood quasars. All these have also been photometrically identified as quasars by Richards et al. (2009), and we list their estimated photometric redshifts in Table A.1 as well, marked by a "P". We note, however, that at $W 1-W 2<0.8$, two spectroscopically confirmed quasars have also been observed, one being a high- $z$ quasar with optical colours consistent with known quasars in this redshift range and the other being a typical UV-excess quasar. These have MIR colours more consistent with typical stellar colours, as also illustrated in Fig. 3. We therefore consider the sources with zero proper motions and $W 1-W 2<0.8$ as possible contaminants (excluding the two already known quasars). We then infer a conservative selection efficiency of $N_{\mathrm{QSO}} / N_{\mathrm{star}} \gtrsim 75 \%$. This is a lower limit due to the population of quasars with blue $W 1-W 2$ colours that also populates our sample.

We present our main result in Fig. 4 where we show the full sample of Gaia sources with $18<G<20 \mathrm{mag}$ and within one degree of the NGP for which a counterpart in the AllWISE catalogue could be identified. It is clear from the figure that the majority of point sources selected on the basis of zero proper motions occupy a distinct region in $\mathrm{S} / \mathrm{N}_{\mu}-$ WISE colour parameter space. This demonstrates that selecting quasars as stationary sources on the sky is definitely feasible and has a high efficiency of $\gtrsim 75 \%$. The completeness is close to $100 \%$ within

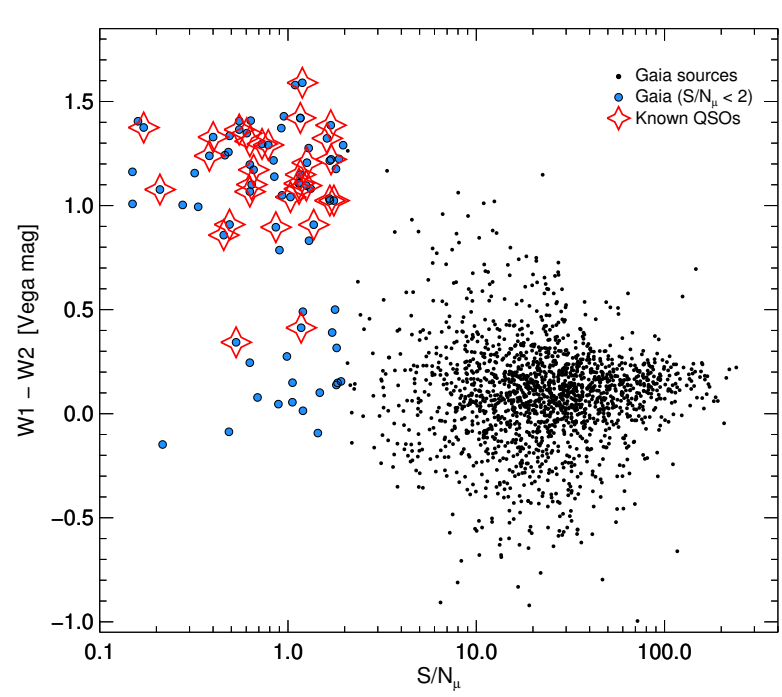

Fig. 4. W1-W2 colour as a function of $\mathrm{S} / \mathrm{N}_{\mu}$ of the WISE-detected Gaia point sources with proper motions and $18<G<20$ mag (black dots) within one degree of the NGP. Gaia point sources with zero proper motions are represented by blue dots and spectroscopically confirmed quasars are shown with red star symbols.

the defined magnitude limit, since all cosmological objects are selected without any prior assumptions on the spectral energy distributions.

\section{Discussion and conclusions}

Here we have demonstrated the possibility to select quasars as stationary objects in the Gaia DR2 data set. When observing fields well away from the Galactic stellar disk (here the NGP) the contamination from stars is very modest (below 25\%) when targeting the most relevant magnitudes (here $18<G<20$ ). Therefore, astrometric selection offers both a complete and clean selection of quasars.

This technique offers the possibility to take major steps ahead on some very interesting problems relating to the quasar phenomenon. We mention a few examples here. First, obtaining a more complete picture of dust obscuration in quasar hosts will be possible with a sample of quasars selected using proper motions. Second, the redshift dependence of the frequency of BAL quasars can be determined. Third, using a purely astrometrically selected sample of quasars, we can get an independent gauge of the metallicity distribution of intervening galaxies, in particular the damped Lyman- $\alpha$ absorbers. Fourth, the identification of quasars via zero proper motion also provides unbiased measurements of number densities of various absorbers, such as $\mathrm{C}$ IV, $\mathrm{Mg}$ II, and $\mathrm{H}$ I. Such a sample will still be subject to a flux limit, but this is easier to model than the combined effect of a flux limit and the effect of dust reddening on the quasar-selection efficiency in optical quasar surveys. We also note that the Gaia DR2 data have been applied to find new gravitationally lensed quasars (Krone-Martins et al. 2018).

An interesting case is the confirmed quasar SDSS $\mathrm{J} 125209.59+265018.4$ (GQ125209+265018 in Table 1). In Fig. 2, this is located as the object on the stellar track at $u-g=1.5$. In Fig. 3, it is one of the two sources with blue WISE colours at $W 1-W 2<0.8$. This illustrates well the capability of the selection of quasars from astrometry to find quasars that are otherwise difficult to photometrically identify. 
Table 1. Point sources within one degree of the NGP with proper motions consistent with zero (within $2 \sigma$ ) and $18<G<20$ mag.

\begin{tabular}{lccccccccc}
\hline \hline Source & RA & Dec & $u$ & $g$ & $r$ & $i$ & $z$ & $W 1-W 2$ & $z_{\text {QSO }}$ \\
\hline GQ125420+274609 & $12: 54: 20.0$ & $+27: 46: 09.6$ & 23.2 & 21.4 & 20.0 & 19.1 & 18.7 & - & - \\
GQ125302+261711 & $12: 53: 02.1$ & $+26: 17: 11.2$ & 20.0 & 19.4 & 19.6 & 19.5 & 19.2 & 0.86 & 2.32 \\
GQ125358+262453 & $12: 53: 58.1$ & $+26: 24: 53.7$ & 20.9 & 20.1 & 19.8 & 19.9 & 19.8 & 0.91 & 3.10 \\
GQ125302+262722 & $12: 53: 02.2$ & $+26: 27: 22.5$ & 19.7 & 19.5 & 19.1 & 19.0 & 19.1 & 1.21 & 1.26 \\
GQ125456+263623 & $12: 54: 56.8$ & $+26: 36: 23.8$ & 23.2 & 20.8 & 19.6 & 19.0 & 18.8 & -0.09 & - \\
\hline
\end{tabular}

Notes. Right ascension and declination are from the Gaia DR2 catalogue. Optical magnitudes are in the AB system and are from the SDSS photometric data. The MIR $W 1-W 2$ colours are from the AllWISE catalogue and are in the Vega system. Spectroscopic redshifts are based on the already identified quasars in the SDSS-DR14 quasar survey (Pâris et al. 2018) or the NED database (specifically from Crampton et al. 1987). Photometric redshifts (marked by a "P") are from Richards et al. (2009). The full table is available in Appendix A.

When the full Gaia data are released, the errors on the proper motions will decrease and it will therefore be easier to disentangle objects that are truly stationary (quasars) from stars with low proper motions. This will also make it possible to search for stationary sources at even fainter magnitudes. Also, since Gaia astrometry exists for most of the sky, this proper motion criteria could help reduce the contamination in other quasar surveys. Since Gaia covers the full sky, the selection can also be carried out for a large sample of sources; however, with the caveat that the contamination from apparently stationary stars increases significantly closer to the Galactic plane. We can also estimate the expected contamination of, for example, the WISE $W 1-W 2$ colour selection, where it can be seen from Fig. 4 that $15 \%$ of the sources with $W 1-W 2>0.8$ have significant proper motions at more than $5 \sigma$.

Acknowledgements. We would like to thank the referee for a clear and constructive report. KEH and PJ acknowledge support by a Project Grant (162948-051) from The Icelandic Research Fund. The Cosmic Dawn Center is funded by the DNRF. LC is supported by DFF - 4090-00079.

\section{References}

Alam, S., Albareti, F. D., Allende Prieto, C., et al. 2015, ApJS, 219, 12 Brusa, M., Civano, F., Comastri, A., et al. 2010, ApJ, 716, 348
Crampton, D., Cowley, A. P., \& Hartwick, F. D. A. 1987, ApJ, 314, 129

Fynbo, J. P. U., Krogager, J.-K., Venemans, B., et al. 2013, ApJS, 204, 6

Gaia Collaboration (Brown, A. G. A., et al.) 2018, A\&A, 616, A1

Heintz, K. E., Fynbo, J. P. U., \& Høg, E. 2015, A\&A, 578, A91

Hewett, P. C., \& Foltz, C. B. 1994, PASP, 106, 113

Ivezić, Ž., Menou, K., Knapp, G. R., et al. 2002, AJ, 124, 2364

Koo, D. C., Kron, R. G., \& Cudworth, K. M. 1986, PASP, 98, 285

Krawczyk, C. M., Richards, G. T., Gallagher, S. C., et al. 2015, AJ, 149 , 203

Krogager, J.-K., Geier, S., Fynbo, J. P. U., et al. 2015, ApJS, 217, 5

Krogager, J.-K., Fynbo, J. P. U., Heintz, K. E., et al. 2016, ApJ, 832, 49

Krone-Martins, A., Delchambre, L., Wertz, O., et al. 2018, A\&A, submitted, [arXiv:1804.11051]

Lindegren, L., Hernandez, J., Bombrun, A., et al. 2018, A\&A, 616, A2

Maddox, N., Hewett, P. C., Péroux, C., Nestor, D. B., \& Wisotzki, L. 2012, MNRAS, 424, 2876

Pâris, I., Petitjean, P., Aubourg, E., et al. 2018, A\&A, 613, A51

Richards, G. T., Myers, A. D., Gray, A. G., et al. 2009, ApJS, 180, 67

Ross, N. P., Hamann, F., Zakamska, N. L., et al. 2015, MNRAS, 453, 3932

Sandage, A. 1965, ApJ, 141, 1560

Schmidt, M., \& Green, R. F. 1983, ApJ, 269, 352

Schneider, D. P., Richards, G. T., Hall, P. B., et al. 2010, AJ, 139, 2360

Secrest, N. J., Dudik, R. P., Dorland, B. N., et al. 2015, ApJS, 221, 12

Stern, D., Assef, R. J., Benford, D. J., et al. 2012, ApJ, 753, 30

Weymann, R. J., Carswell, R. F., \& Smith, M. G. 1981, ARA\&A, 19, 41

Wright, E. L., Eisenhardt, P. R. M., Mainzer, A. K., et al. 2010, AJ, 140, 1868 
K. E. Heintz et al.: Unidentified quasars among stationary objects from Gaia DR2

\section{Appendix A: Table}

Table A.1. Point sources within one degree of the NGP with proper motions consistent with zero (within $2 \sigma$ ) and $18<G<20$ mag.

\begin{tabular}{|c|c|c|c|c|c|c|c|c|c|}
\hline Source & RA & Dec & $u$ & $g$ & $r$ & $i$ & $z$ & $W 1-W 2$ & $z_{\mathrm{QSO}}$ \\
\hline $20+274609$ & $: 54: 20.0$ & $27: 46: 09.6$ & 23.2 & 21.4 & 20.0 & 19.1 & 18.7 & - & - \\
\hline 711 & $12: 53$ & $+26: 1$ & 20.0 & 19.4 & & & & & 2.32 \\
\hline GQ125358+262453 & $12: 53: 58.1$ & $+26: 24: 53.7$ & 20.9 & 20.1 & 19.8 & 19.9 & 19.8 & 0.91 & 3.10 \\
\hline GQ125302+262722 & $12: 53: 02.2$ & $+26: 27: 22.5$ & 19.7 & 19.5 & 19.1 & 19.0 & 19.1 & 1.21 & 1.26 \\
\hline GQ125456+263623 & $12: 54: 56.8$ & $+26: 36: 23.8$ & 23.2 & 20.8 & 19.6 & 19.0 & 18.8 & -0.09 & - \\
\hline GQ125510+263724 & $12: 55: 10.1$ & $+26: 37: 25.0$ & 21.0 & 20.2 & 19.9 & 19.9 & 19.8 & - & - \\
\hline GQ125424+263941 & $12: 54: 24.3$ & $+26: 39: 41.8$ & 19.3 & 19.1 & 19.2 & 19.0 & 19.1 & 1.59 & 1.60 \\
\hline GQ125453+263721 & $12: 54: 53.0$ & $+26: 37: 21.8$ & 20.9 & 19.8 & 19.5 & 19.4 & 19.2 & - & - \\
\hline GQ125503+264502 & $12: 55: 03.8$ & $+26: 45: 02.7$ & 21.3 & 20.2 & 19.6 & 19.4 & 19.2 & - & - \\
\hline GQ125441+264637 & $12: 54: 41.3$ & $+26: 46: 37.8$ & 20.9 & 19.7 & 19.3 & 19.0 & 19.0 & - & - \\
\hline $424+264833$ & $12: 54: 24.6$ & $+26: 48: 33.8$ & 20.1 & 19.9 & 19.7 & 19.6 & 19.7 & 1.22 & $1.47 \mathrm{P}$ \\
\hline $16+265049$ & $12: 55: 16.1$ & $+26: 50: 49.0$ & 25.0 & 21.1 & 19.8 & 19.2 & & - & - \\
\hline 034 & 12: & $+27: 0$ & 23.9 & 21.2 & 19.7 & 18.2 & 17.5 & 0.16 & - \\
\hline GQ12 & $12: 55: 43.7$ & $+27: 07: 14.1$ & 23.8 & 21.0 & 19.7 & 18.2 & 17.5 & 0.14 & star \\
\hline GQ1 & $12: 51: 26.6$ & $+26: 13: 46.9$ & 19.6 & 19.7 & 19.4 & 19.3 & 19.4 & 1.11 & 1.43 \\
\hline 629 & 12:49: & $+26: 1$ & 18.9 & & 18.7 & 18.5 & 18 & 0.34 & 1.84 \\
\hline 49 & $12: 49$ & $+26: 1$ & 21.1 & 20 & 19.9 & 19.7 & 19.7 & - & - \\
\hline 953 & $12: 50: 31.6$ & $+26: 1 \mathrm{C}$ & 19.6 & 19.4 & 19.3 & 19.4 & 19.4 & 1.00 & $0.88 \mathrm{P}$ \\
\hline $27+263048$ & $12: 50: 27.3$ & $+26: 30$ : & 20.7 & 19.5 & 19.0 & 18.8 & 18.7 & - & - \\
\hline $48+263805$ & $12: 52: 48.3$ & $+26: 38: 05.6$ & 24.8 & 21.6 & 20 & 19.0 & & 0.08 & - \\
\hline 3900 & & $+26: 3$ & 17.7 & 17.6 & & & & & 1.91 \\
\hline 550 & $12: 5$ & $+26:$ & 20.0 & 19.9 & 19.4 & 19.4 & 19 & 1.42 & $1.28 \mathrm{P}$ \\
\hline $209+265018$ & $12: 52: 09.6$ & $+26: 50: 18.5$ & 21.9 & 20.4 & 19.7 & 19.5 & 19 & 0.41 & 3.44 \\
\hline $902+262014$ & $12: 49: 02.2$ & $+26: 20:$ & 22.6 & 20.8 & 19.7 & 19.1 & 18 & - & - \\
\hline & & & & & & & & $1.0 \mathrm{~J}$ & $0.68 \mathrm{P}$ \\
\hline 759 & $12: 48$ & $+26: 3$ & 20.1 & 20.1 & 19.9 & 19.8 & 19.9 & 1.28 & $1.15 \mathrm{P}$ \\
\hline GQ124820+264225 & $12: 48: 20.6$ & $+26: 42$ & 20.4 & & 19.4 & 19.4 & 19.6 & - & - \\
\hline GQ124926+264101 & $12: 49: 26.1$ & $+26: 41: 01.4$ & 19.7 & 19.3 & 19.2 & 19.2 & 19.2 & 1.10 & 0.67 \\
\hline & $12: 49: 21.2$ & $+26: 44: 46.8$ & & & & & & & $0.31 \mathrm{P}$ \\
\hline & & & & & & & & & $1.59 \mathrm{P}$ \\
\hline+264337 & $12: 50$ & $+26: 4$ & 18.5 & & & 18.2 & 18 & 1.38 & 1.79 \\
\hline GQ125149+265349 & $12: 51: 49.2$ & $+26: 53: 50.0$ & 23.1 & 21.0 & 19.8 & 19.3 & 19.1 & 0.50 & star \\
\hline GQ124945+264953 & $12: 49: 45.5$ & $+26: 49: 53.3$ & 25.0 & & 19.8 & 19.1 & 18.8 & 0.25 & - \\
\hline & & & & & & & & - & $2.46 \mathrm{P}$ \\
\hline 28 & 12:50: & $+26: 5$ & 19.8 & 19.4 & 19.0 & 18.9 & 18.9 & 1.08 & 1.49 \\
\hline GQ124935+265906 & $12: 49: 35.2$ & $+26: 59: 06.4$ & 25.0 & 21.4 & 19.9 & 19.1 & 18.7 & 0.15 & - \\
\hline GQ125006+265939 & $12: 50: 06.3$ & $+26: 59: 39.9$ & 19.9 & 19.8 & 19.5 & 19.7 & 19.7 & 1.20 & 0.97 \\
\hline GQ125421+265454 & $12: 54: 21.0$ & $+26: 54: 54.7$ & 20.2 & & & & 19.6 & 1.16 & $1.30 \mathrm{P}$ \\
\hline & & & & & & & & 1.10 & 1.64 \\
\hline $312+265355$ & $12: 53: 12.1$ & $+26: 53: 55.7$ & 24.6 & 21.5 & 20.0 & 18.9 & 18.4 & - & - \\
\hline GQ125302+270519 & $12: 53: 02.3$ & $+27: 05: 19.9$ & 22.6 & 20.4 & 20.1 & 20.0 & 20.0 & 1.02 & 2.99 \\
\hline GQ125320+270607 & $12: 53: 20.1$ & $+27: 06: 07.4$ & 20.1 & 19.7 & 19.1 & 18.8 & 18.9 & 1.32 & 1.15 \\
\hline GQ125528+271330 & $12: 55: 28.8$ & $+27: 13: 30.1$ & 20.0 & 19.8 & 19.5 & 19.7 & 19.7 & 1.30 & 1.03 \\
\hline GQ125357+271630 & $12: 53: 57.5$ & $+27: 16: 30.6$ & 22.2 & 19.9 & 18.9 & 18.5 & 18.3 & - & - \\
\hline GQ125359+272014 & $12: 53: 59.8$ & $+27: 20: 14.8$ & 20.6 & 20.3 & 20.3 & 19.9 & 20.4 & 1.58 & $0.08 \mathrm{P}$ \\
\hline GQ125158+271304 & $12: 51: 58.6$ & $+27: 13: 04.1$ & 21.6 & 20.2 & 19.7 & 19.5 & 19.6 & - & - \\
\hline & $12: 53: 00.1$ & $+27: 12: 34.2$ & 19.5 & & 19.1 & 19.0 & & 1.42 & 1.48 \\
\hline GQ125241+272550 & $12: 52: 41.1$ & $+27: 25: 50.8$ & 21.4 & 21.2 & 20.7 & 20.1 & 19.9 & 1.22 & $0.22 \mathrm{P}$ \\
\hline
\end{tabular}

Notes. Right ascension and declination are from the Gaia DR2 catalogue. Optical magnitudes are in the AB system and are from the SDSS photometric data. The mid-infrared $W 1-W 2$ colours are from the AllWISE catalogue and are in the Vega system. Spectroscopic redshifts are based on the already identified quasars in the SDSS-DR14 quasar survey (Pâris et al. 2018) or the NED database (specifically from Crampton et al. 1987). Photometric redshifts (marked by a "P") are from Richards et al. (2009). 
A\&A 615, L8 (2018)

Table A.1. continued.

\begin{tabular}{|c|c|c|c|c|c|c|c|c|c|}
\hline Source & RA & Dec & $u$ & $g$ & $r$ & $i$ & $z$ & $W 1-W 2$ & $z_{\mathrm{QSO}}$ \\
\hline GQ125134+272000 & 12.51 .54 .4 & $+27: 20: 00.2$ & 20.9 & & 19.7 & 19.7 & & - & 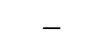 \\
\hline $107+271451$ & $12: 51: 08.0$ & $+27: 14: 51.5$ & 20.2 & 19.8 & 19.4 & 19.2 & 18.8 & 0.79 & $0.38 \mathrm{P}$ \\
\hline GQ125206+272717 & $12: 52: 07.0$ & $+27: 27: 17.5$ & 18.7 & 18.6 & 18.6 & 18.4 & 18.5 & 1.22 & 1.68 \\
\hline GQ125211+272803 & $12: 52: 11.9$ & $+27: 28: 03.8$ & 20.7 & 20.6 & 20.4 & 20.0 & 20.1 & 1.41 & $1.87 \mathrm{P}$ \\
\hline GQ125332+272225 & $12: 53: 32.1$ & $+27: 22: 25.1$ & & & & & & & 1.63 \\
\hline GQ125320+272116 & $12: 53: 20.3$ & $+27: 21: 16.0$ & 18.0 & 17.8 & 18.0 & 17.9 & 17.8 & & 0.51 \\
\hline GQ125353+273405 & $12: 53: 53.7$ & $+27: 34: 05.9$ & 21.4 & 20.3 & 19.8 & 19.5 & 19.5 & & - \\
\hline GQ125308+273331 & $12: 53: 08.5$ & $+27: 33: 31.2$ & 23.8 & 21.6 & 20.4 & 19.2 & 18.5 & -0.09 & - \\
\hline GQ125327+273733 & $12: 53: 27.1$ & $+27: 37: 33.1$ & 19.9 & 19.9 & 19.8 & 19.6 & 19.4 & & $1.96 \mathrm{P}$ \\
\hline $7+274542$ & $12: 52: 58.0$ & $+27: 45:$ & 19.0 & 18 & 18.6 & 18.5 & 18.4 & 1.32 & 2.00 \\
\hline $6+264712$ & $12: 48: 16.3$ & $+26: 47:$ & 18.1 & & 18.2 & 17.9 & 17.8 & 1.35 & 1.86 \\
\hline GQ12 & $12: 47: 46.5$ & $+26: 47: 09.8$ & 21.2 & 20.2 & 20.0 & 19.8 & 19.7 & - & - \\
\hline GQ12 & 12:48: & $+26: 52: 35.3$ & 20.1 & 19.5 & 19.4 & 19.4 & 19.3 & - & 2.51 \\
\hline $32+265312$ & $12: 48: 32.8$ & $+26: 53: 12.7$ & 18.2 & 17 & 17.9 & 17.7 & 17 & 1.11 & 0.59 \\
\hline $4+265836$ & $12: 48: 05.0$ & $+26: 58: 36.1$ & 24.8 & 2 & 20 & 19.1 & & 0.15 & - \\
\hline 28 & & +26 & 22.4 & & & & & - & - \\
\hline 728 & $12: 47: 21.3$ & $+26: 57: 2$ & 21.3 & 20.2 & 19.7 & 19.5 & 19.4 & - & - \\
\hline GQ1 & $12: 47: 25.8$ & $+27: 01: 14.1$ & 19.8 & 19.3 & 19.2 & 19.2 & 19.0 & 1.07 & 0.80 \\
\hline 22 & $12: 47$ & $+27: 06$ & 20.9 & 20 & 19 & 19.7 & 1 & - & - \\
\hline & & $+27: 06$ & 23.7 & & & 19.0 & & 0.05 & - \\
\hline & & $+26: 5$ & 21.3 & & & 19.5 & & - & - \\
\hline $5+271335$ & $12: 49$ & $+27: 13:$ & 19.5 & 19.4 & 19 & 19.2 & 19 & 1.37 & $1.47 \mathrm{P}$ \\
\hline $5+271722$ & 12:48: & $+27: 17: 22.7$ & 19.8 & 19 & 19 & 18.8 & & 1. & 1.51 \\
\hline 603 & & $+27: 16$ & 22.8 & & & 18.6 & & & - \\
\hline & .4 & & 20.2 & & & 19.8 & 19 & & $0.95 \mathrm{P}$ \\
\hline GQ124925+272634 & $12: 49$ & $+27: 26:$ & 18.7 & 18.5 & 18 & 18.4 & 18.5 & 1.37 & 1.16 \\
\hline GQ124728+272742 & $12: 47$ & $+27: 27: 42.5$ & 21.2 & & 20 & 19.9 & & - & $0.46 \mathrm{P}$ \\
\hline 3018 & & $+27: 30: 18.9$ & & & & & & 1 & 0.91 \\
\hline 934 & $12: 5$ & $+27: 19$ & 20.3 & 20.2 & 20.1 & 20.2 & 20.0 & 0.83 & $0.93 \mathrm{P}$ \\
\hline GQ125050+272448 & $12: 50$ & $+27: 24: 48.4$ & 24.6 & 21.5 & 20.1 & 18.7 & 17.9 & & - \\
\hline GQ125039+272904 & $12: 50: 39.4$ & $+27: 29: 04.4$ & 20.7 & 20.1 & 19.6 & 19.1 & 18.9 & 1. & 1.89 \\
\hline & & $+27: 33: 41.2$ & 20.2 & & & 19.8 & & & $1.15 \mathrm{P}$ \\
\hline & & & & & & & & & $1.72 \mathrm{P}$ \\
\hline 256 & .7 & $+27: 3$ & 19.9 & 19.8 & 19 & 19.5 & 19.7 & 1.14 & $1.15 \mathrm{P}$ \\
\hline GQ125008+274001 & $12: 50: 08.6$ & $+27: 40: 01.5$ & 21.1 & 20.1 & 19.9 & 19.7 & 19.8 & 0.32 & - \\
\hline GQ125042+274012 & $12: 50: 42.4$ & $+27: 40: 13.0$ & 20.2 & 20.0 & 19.7 & 19.6 & 19.6 & 1.41 & $1.48 \mathrm{P}$ \\
\hline & & $+27: 45: 19.1$ & 20.0 & & & 19.6 & & 1. & $1.48 \mathrm{P}$ \\
\hline 4443 & $12: 50: 19.2$ & $+27: 44:$ & 20.5 & 19.7 & 19.4 & 19.1 & 18.7 & 1.02 & 2.67 \\
\hline $7+274709$ & $12: 50: 07.9$ & $+27: 47: 09.4$ & 23.6 & 21.4 & 19.8 & 18.7 & 18.0 & & - \\
\hline GQ125202+275018 & $12: 52: 02.1$ & $+27: 50: 18.6$ & 19.3 & 18.8 & 18.7 & 18.7 & 18.5 & 1.17 & 2.48 \\
\hline GQ125312+275524 & $12: 53: 12.1$ & $+27: 55: 24.6$ & 19.7 & & & 19.3 & & 1.18 & $1.00 \mathrm{P}$ \\
\hline & & & & & & & & & $1.04 \mathrm{P}$ \\
\hline GQ125241+275942 & $12: 52: 42.0$ & $+27: 59: 42.7$ & 24.0 & 21.8 & 20.6 & 19.1 & 18.3 & 0.10 & - \\
\hline GQ125239+280127 & $12: 52: 39.2$ & $+28: 01: 27.2$ & 19.6 & 19.6 & 19.5 & 19.4 & 19.2 & 1.29 & $2.04 \mathrm{P}$ \\
\hline GQ125249+280356 & $12: 52: 49.1$ & $+28: 03: 56.4$ & 19.4 & 19.3 & 19.2 & 19.4 & 19.2 & 1.01 & $0.93 \mathrm{P}$ \\
\hline GQ125112+275222 & $12: 51: 12.3$ & $+27: 52: 22.4$ & 19.0 & 18.8 & 18.5 & 18.5 & 18.6 & 1.24 & 1.04 \\
\hline GQ125159+280446 & $12: 51: 59.1$ & $+28: 04: 46.3$ & 23.1 & 21.4 & 19.9 & 18.8 & 18.2 & 0.01 & - \\
\hline GQ124901+274330 & 12:49:01.8 & $+27: 43: 30.2$ & 21.1 & 20.2 & 19.8 & 19.7 & 19.5 & - & - \\
\hline GQ124915+274554 & $12: 49: 15.9$ & $+27: 45: 54.8$ & 19.6 & 19.3 & 19.3 & 19.2 & 18.9 & 1.43 & $2.16 \mathrm{P}$ \\
\hline GQ12495 & $12: 49: 55.4$ & $+27: 56: 57.7$ & 21.2 & & & 19.0 & 18.9 & 0.49 & - \\
\hline GQ124958+275703 & $12: 49: 58.1$ & $+27: 57: 03.7$ & 19.8 & 19.6 & 19.4 & 19.1 & 19.0 & 1.34 & $1.91 \mathrm{P}$ \\
\hline GQ125005+280041 & $12: 50: 05.2$ & $+28: 00: 41.9$ & 21.0 & 20.2 & 20.0 & 19.8 & 19.7 & - & - \\
\hline
\end{tabular}


K. E. Heintz et al.: Unidentified quasars among stationary objects from Gaia DR2

Appendix B: Thumbnail images of all sources

Thumbnails of all point sources within one degree of the NGP with proper motions consistent with zero (within
$2 \sigma)$ and $18<G<20 \mathrm{mag}$. East is up and north is to the right.
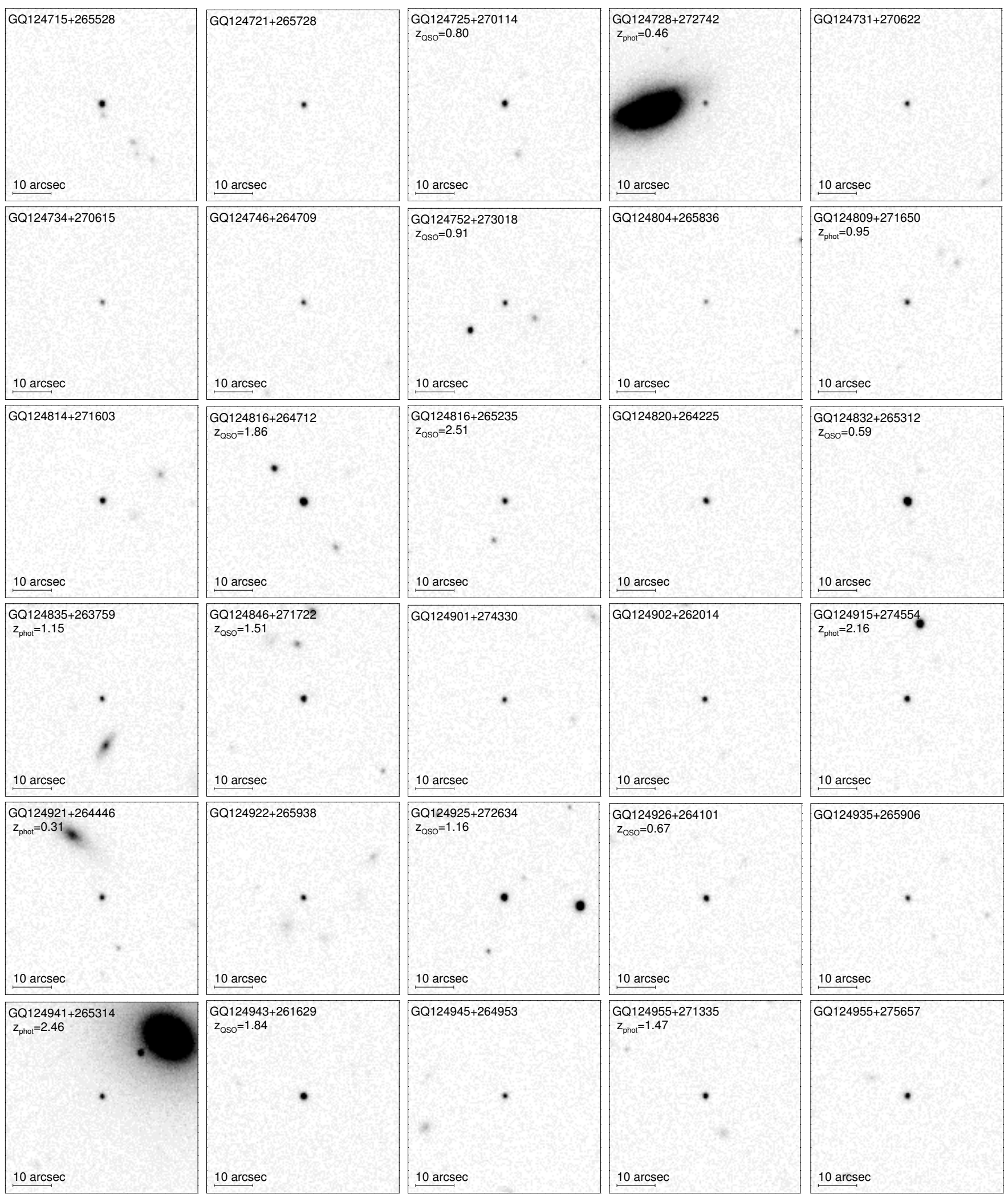

GQ124804+265836

GQ124809+271650

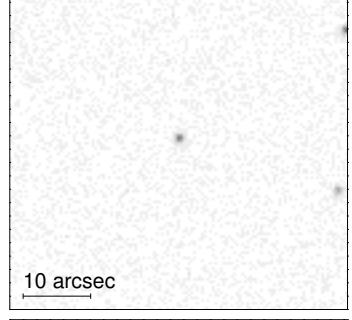

$\mathrm{z}_{\text {phot }}=0.95$

GQ124816+264712

GQ124816+265235

GQ124820+264225

10 arcsec

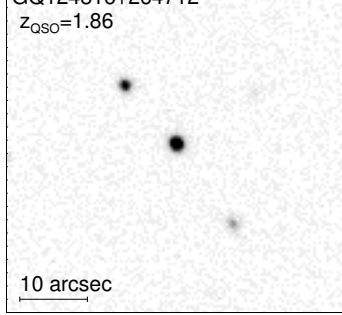

$\mathrm{z}_{\mathrm{QSO}}=2.51$

GQ124832+265312

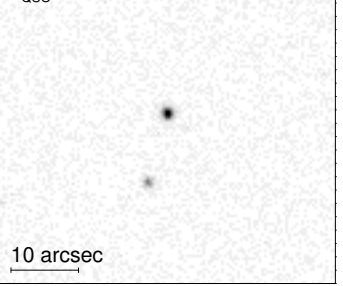

GQ124846+271722

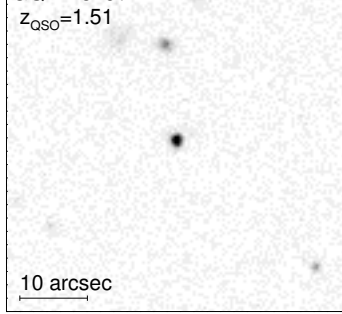

GQ124901+274330

10 arcsec

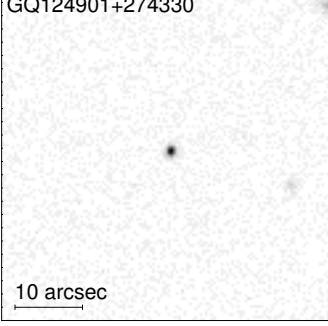

GQ124902+262014

$10 \operatorname{arcsec}$
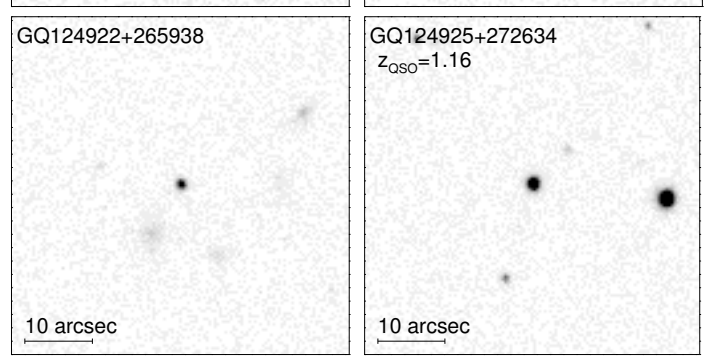

$10 \operatorname{arcsec}$

GQ124926+264101

$10 \operatorname{arcsec}$

$\mathrm{z}_{\mathrm{OSO}}=0.67$

GQ124943+261629

GQ124945+264953

$10 \operatorname{arcsec}$

$\mathrm{z}_{\mathrm{OSO}}=1.84$

GQ124955+271335

$Z_{\text {phot }}=1.47$
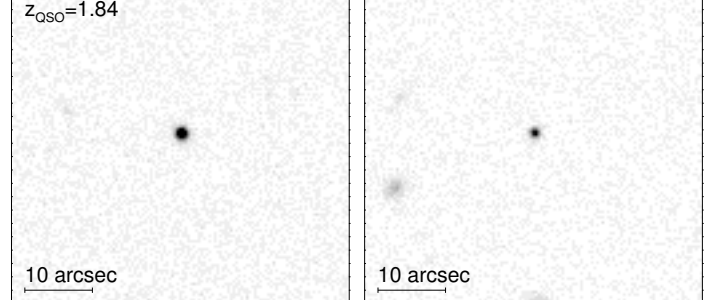

Fig. B.1. $50 \times 50 \operatorname{arcsec}^{2}$ thumbnails around each stationary source. 
A\&A 615, L8 (2018)

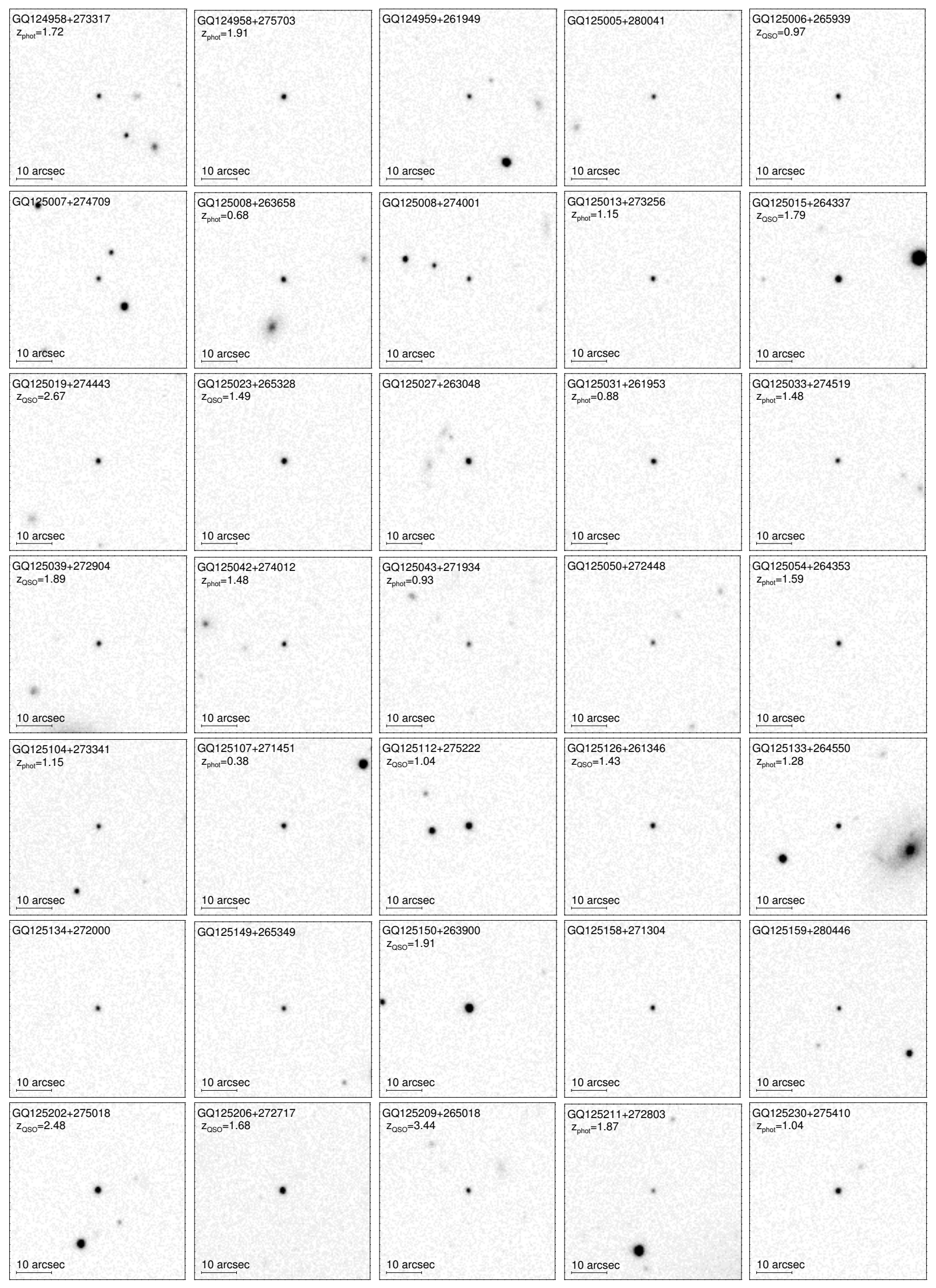

Fig. B.1. continued. 
K. E. Heintz et al.: Unidentified quasars among stationary objects from Gaia DR2

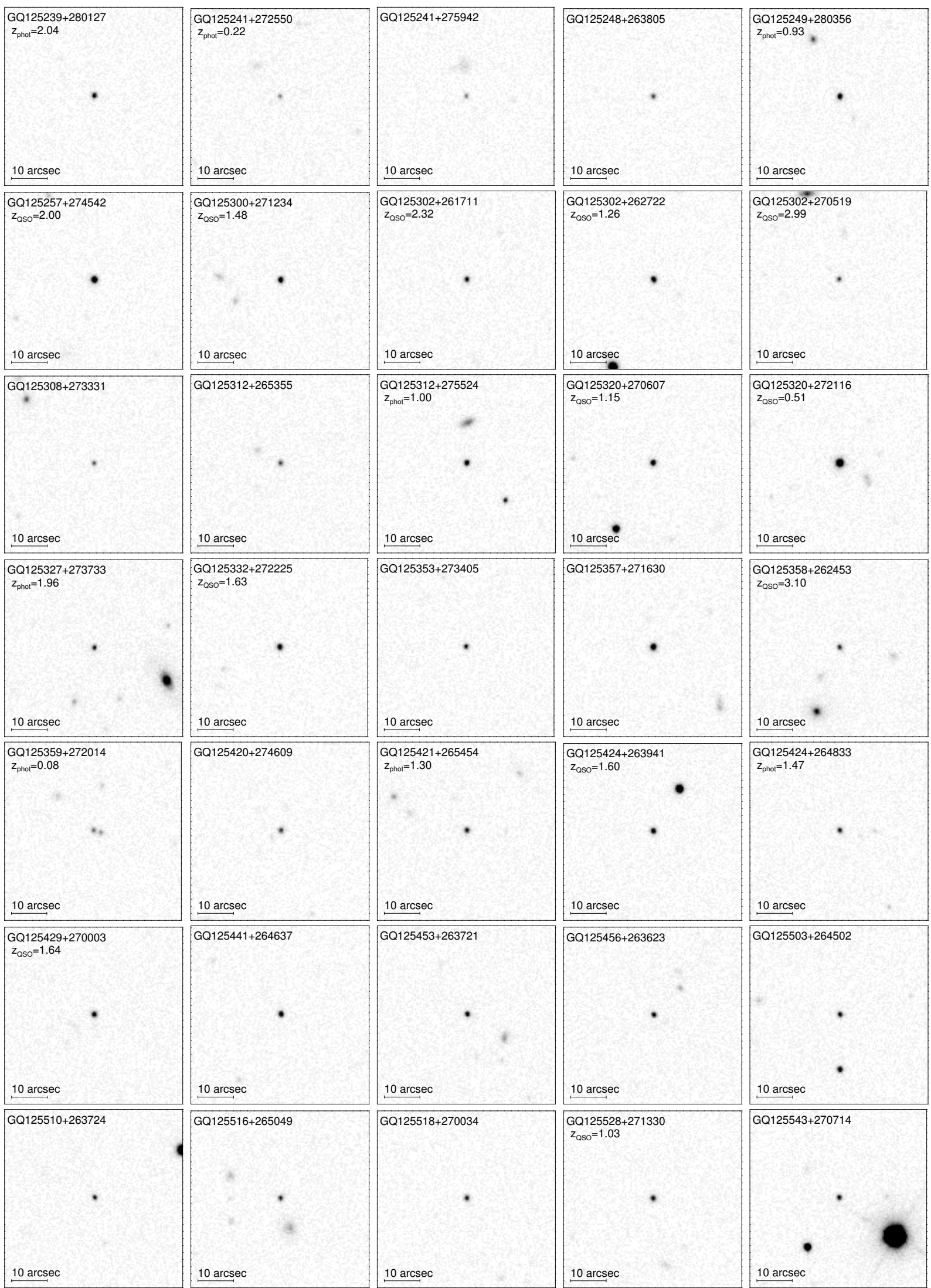

Fig. B.1. continued. 